\title{
Psychotropic drug utilization in psychiatric outpatient department of a tertiary care government hospital
}

\author{
Pragyan Paramita Jena ${ }^{1}$, Birendra Narayan Naik ${ }^{2 *}$, Bhabagrahi Rath ${ }^{1}$
}

${ }^{1}$ Department of Pharmacology, ${ }^{2}$ Department of Psychiatry, VIMSAR, Burla, Sambalpur, Odisha, India

\author{
Received: 01 February 2020 \\ Revised: 15 April 2020 \\ Accepted: 16 April 2020 \\ *Correspondence: \\ Dr. Birendra Narayan Naik, \\ Email: Pragyanjena41@gmail.com
}

Copyright: $@$ the author(s), publisher and licensee Medip Academy. This is an open-access article distributed under the terms of the Creative Commons Attribution Non-Commercial License, which permits unrestricted non-commercial use, distribution, and reproduction in any medium, provided the original work is properly cited.

\begin{abstract}
Background: Mental disorders are associated with significant distress in social, occupational and other important activities. Of the ten health conditions contributing to the DALYs, four are psychiatric disorders. Psychiatric disorders form an important public health priority and major causes of morbidity. Psychotropic drugs had a remarkable impact in psychiatric practice. The utilization, clinical practice, effectiveness and safety in real life situation need continuous study.

Methods: A cross-sectional study was carried out by analyzing prescriptions of patients who visited the OPD of the psychiatry department of VIMSAR, Burla for a period of 4 months. Patients of all ages and both sexes were included in this study with exclusion of patients suffering from epilepsy and cases with uncertain diagnosis. The prescribing pattern was analyzed using WHO basic drug indicators.

Results: Average number of drugs per prescription was 3.17. Bipolar affective disorder (BPAD) was the most common psychiatric condition (44\%) with a male predominance followed by schizophrenia. Olanzapine was the most common antipsychotic used. The most frequently used fixed dose combination was tri-hexiphenydyl and risperidone. Prescribing frequency of different psychotropic drugs (antipsychotics, antianxiety, selective serotonin reuptake inhibitors, tricyclic antidepressant, mood stabilizers) were compared.

Conclusions: The study advocated a rational use of psychotropic drugs with fewer deviations due to socio-economic status of patients and prescription practices of healthcare providers.
\end{abstract}

Keywords: Psychotropic drugs, Prescribing pattern, WHO indicators

\section{INTRODUCTION}

Mental disorders are usually associated with significant distress in social, occupational and other important activities. ${ }^{1}$ Of the top ten health conditions contributing to the DALYs, four are pyshiatric disorders. ${ }^{2}$ Psychiatric disorders form an important public health priority and major causes of morbidity. ${ }^{3}$ Drug utilization study has been defined by the WHO as the marketing, distribution, prescription and uses of drugs in a society with special emphasis on the resulting medical, social and economic consequences. ${ }^{4}$ The rationality of prescribing pattern is of utmost importance because, bad prescribing habits includes misuse, overuse and underuse of medicines which can lead to unsafe treatment, exacerbation of the disease, health hazards, economic burden on the patients and wastage of resources. ${ }^{5}$

Drug utilization studies are a pre-requisite for the formulation of drug policies. This identifies the problems that arises from drug usage in health care delivery system and highlights the current approaches to the rational use of drugs. Measurement of drug use in health facilities not only describes drug use patterns and prescribing 
behaviour but also helps in identification of factors responsible for the practice of polypharmacy and the problems associated with it. Setting standards and assessing the quality of care through performance review should become part of everyday clinical practice. ${ }^{6}$ Keeping this in mind, present study was conducted to identify the drugs used in various psychiatric disorders and find out differences between the actual and the ideal prescribing pattern of psychotropic drugs and to estimate the prevalence of various psychiatric illnesses.

\section{METHODS}

The study was conducted after approval issued by Institutional Ethics Commitee, VIMSAR (19-I-S-O149/148).

\section{Study protocol}

This is a cross sectional study conducted by Department of Psychiatry and Pharmacology, VIMSAR, Burla for a period of 4 months (June 2019 to September 2019).

\section{Study sample}

A total of 150 prescriptions was collected at the OPD of Psychiatry department by convenience sampling method.

\section{Inclusion criteria}

Inclusion criteria were patients suffering from any psychiatric illness was included in the study.

\section{Exclusion criteria}

Exclusion criteria were patients suffering from epilepsy, in-patients and those with uncertain diagnosis were excluded from the study.

\section{Data collection and analysis}

The data of total 150 prescriptions was collected and entered into microsoft excel sheet and analysed using
WHO core drug prescribing indicators. The results are expressed in numbers and percentage.

\section{RESULTS}

Total number of drugs prescribed in 150 prescriptions was 476. Average number of psychotropic drugs per encounter was 3.17. Average number of drugs prescribed by generic name was $40.33 \%$. Only 4 prescriptions were found to have injections prescribed. Percentage of encounters with an injection prescribed was $2.66 \%$. Out of total 476 drugs, 236 drugs were found from the essential list of medicines that is $49.57 \%$. Tabular representation of the prescribing indicators is given in (Table 1).

Out of 150 cases reviewed $66(44 \%)$ cases were of bipolar affective disorder (BPAD), the study showed a higher incidence of psychiatric illness in male $(65.15 \%)$ and maximum patients were under the age group of 31-40 years.

Patients of BPAD and schizophrenia accounted for a large majority (about 76\%) of the patients attending the psychiatry OPD in our study followed by acute transient psychotic disorders $(8.7 \%)$.

The most commonly prescribed fixed dose combinations (FDCs) were tri-hexyphenydyl combination with risperidone $(12 \%)$.

Table 1: WHO core drug prescribing indicators.

\begin{tabular}{|ll|}
\hline Indicator & Result \\
\hline $\begin{array}{l}\text { Average number psychotropic of } \\
\text { drugs per encounter }\end{array}$ & 3.17 \\
\hline $\begin{array}{l}\text { Percentage of drugs prescribed by } \\
\text { generic name }\end{array}$ & 40.33 \\
\hline $\begin{array}{l}\text { Percentage of encounters with an } \\
\text { injection }\end{array}$ & 2.66 \\
\hline $\begin{array}{l}\text { Percentage of drugs prescribed from } \\
\text { essential drugs list }\end{array}$ & 49.57 \\
\hline Percentage of drugs used as FDCs & 13.44 \\
\hline
\end{tabular}

Table 2: Morbidity pattern and sex difference among different psychiatric illnesses.

\begin{tabular}{|llll|}
\hline Psychiatry disorder & Male $(\mathbf{n = 9 6 )}$ & Female $(\mathbf{n = 5 4 )}$ & Total (n=150) \\
\hline BPAD & $\mathbf{N}(\mathbf{\%})$ & $\mathbf{N}(\mathbf{\%})$ & 66 \\
\hline Schizophrenia & $43(65.15)$ & $23(34.85)$ & 48 \\
\hline $\begin{array}{l}\text { Acute and transient psychotic } \\
\text { disorder }\end{array}$ & $32(66.67)$ & $16(44.44)$ & 13 \\
\hline Psychosis NOS & $9(69)$ & $4(31)$ & 8 \\
\hline Obsessive compulsive disorder & $4(37.50)$ & $5(62.50)$ & 6 \\
\hline Substance abuse & $2(67)$ & $2(33)$ & 3 \\
\hline Somatoform disorder & $1(50)$ & $1(33)$ & 2 \\
\hline Generalised anxiety disorder & $2(67)$ & $1(50)$ & 3 \\
\hline Depression & 0 & $1(33)$ & 1 \\
\hline
\end{tabular}


Antipsychotic drugs were the most frequent class of drugs prescribed $(27.60 \%)$ followed by anti-anxiety drugs $(26.40 \%)$.

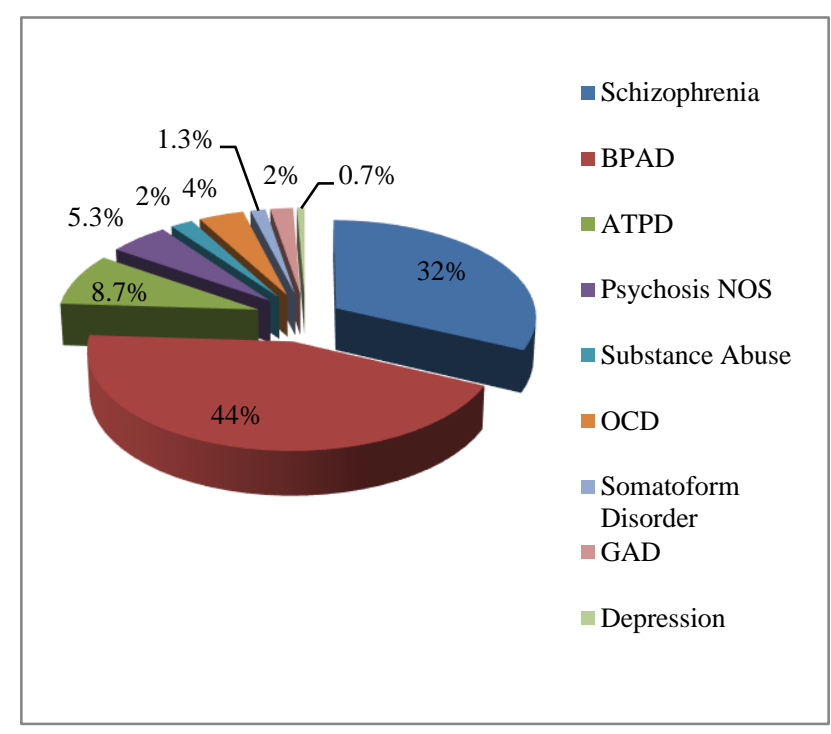

Figure 1: Percentage distribution of various psychiatric illnesses.

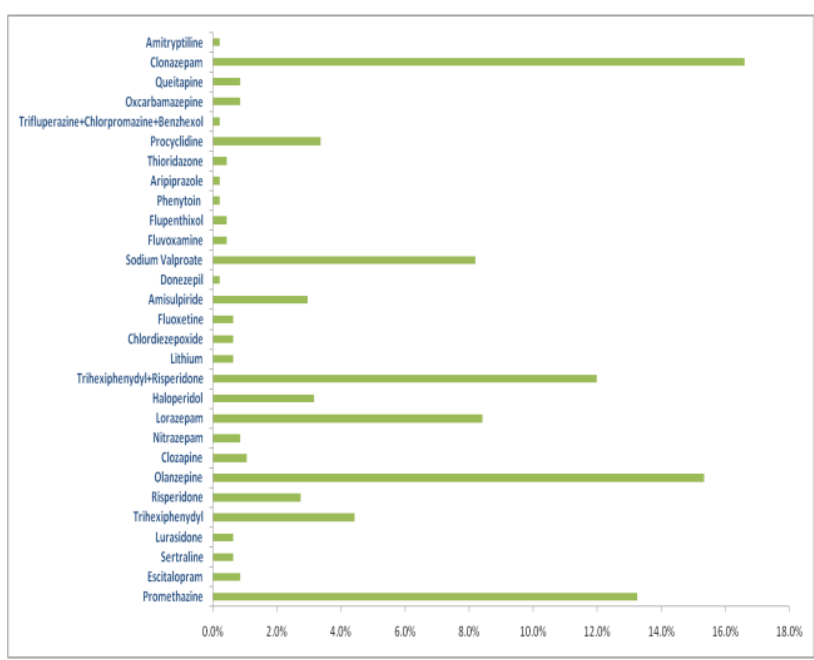

Figure 2: Percentage of drug utilization in psychiatric disorders $(\mathbf{n}=\mathbf{1 5 0})$.

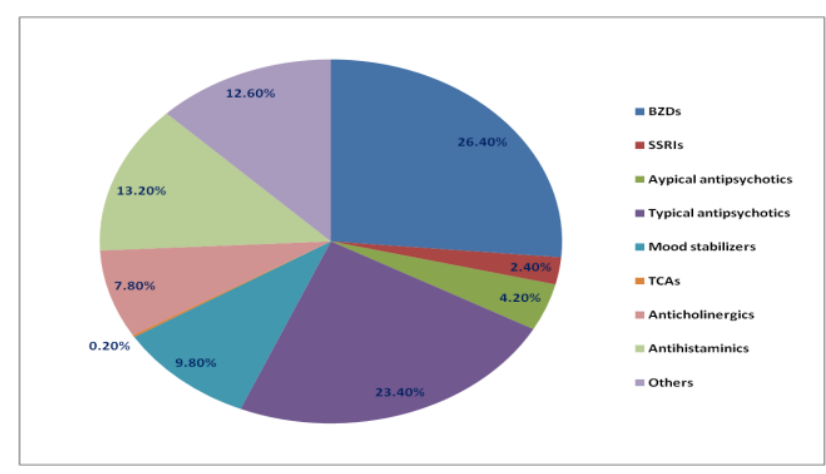

Figure 3: Percentage distribution of various drug class in psychiatry conditions.

\section{DISCUSSION}

The study was carried out to find morbidity pattern of psychiatric diseases and prescribing trends of psychotropic drugs. The study of prescribing patterns seeks to monitor, evaluate and if necessary, suggest modifications in prescribing patterns so as to make medical care rational and cost effective.

The percentage of male patients on medical treatment for psychiatric illness was higher than female patients $(64 \%$ vs $36 \%$ ). Nearly similar results were also observed in a study carried out in OPD of psychiatry at tertiary care teaching hospital in Bastar region $(68.18 \%$ males vs $31.8 \%$ females). ${ }^{7}$ The procreant age group of $21-30$ years reported for the majority of all psychiatric disorder findings of which are similar to studies presented at Mumbai and Jamnagar, India., ${ }^{8,9}$ Increasing use of psychotropic drugs in this age group may be due to increased incidence of mental ill health, improved literacy in general population, reduction in stigma associated with mental illness, increase in drug treatment option and due to more vigorous marketing of such agents. ${ }^{10}$

In the present study the average number of psychotropic drugs per prescription was $3.17 \%$ (which is a reflection of polypharmacy); results of which are similar to studies conducted by Goyal et al, Ajmer. ${ }^{11}$ Oral formulations accounted for $97.34 \%$ and parenteral preparations for remaining $2.66 \%$. This may be due to the fact that most of the patients attending psychiatry OPD belongs to adult age group and can take oral formulations safely and conveniently.

Percentage of drugs prescribed by generic name was $40.33 \%$ which is higher than study conducted in Jalna, Maharastra. ${ }^{12}$ Prescribing medicines by generic name avoid the confusion and makes therapy rational and cheaper. Despite, most doctors prescribe the medicines by their brand names. The reason for this could be tradition, aggressive medicine promotion, availability of multi-ingredient fixed dose drug combination. ${ }^{13}$

Encouraging prescriptions by generic name is always recommended by various national and international bodies to promote rational use of drugs. ${ }^{14}$ It also gives an advantage to the pharmacist to dispense cheaper drugs to the patients, this helps to reduce economic burden on the patients. Also, this practice will certainly help to check the luring practices if offered by some of the pharmaceutical companies to the practitioners for promoting their costlier brands. But implementation of this practice of prescribing by generic names is not always satisfactory and requires motivation of prescribers and strong regulatory interventions. ${ }^{14}$

The most commonly prescribed FDCs were trihexyphenydyl combination with risperidone (12\%) in 
management of schizophrenia, findings of which is similar to study reported in Anantapur, Andhra Pradesh. ${ }^{15}$

Anticholinergic agents are recommended to avoid EPS associated with use of typical (classical) antipsychotics. 'A study of atypical antipsychotic use for adult outpatients' by Wheeler. observed that the anticholinergic drug was used either with atypical antipsychotic or with prescriptions containing both typical and atypical antipsychotic drugs together. However, this study also revealed that co-prescribing of anticholinergic drug may add to new or additive adverse effects (e.g., dry mouth, blurred vision, constipation), which further reduces the quality of life. ${ }^{16}$ As routine use of anticholinergic agents add to the complexity, side effects, and expenses, whether they should be prescribe routinely or reserved for the cases of overt EPS remains open to question.

Antipsychotic drugs were the most frequent class of drugs prescribed $(27.60 \%)$ followed by anti-anxiety drugs $(26.40 \%)$.

The benzodiazepine group was the most frequently prescribed group of anti-anxiety drugs. Further among all anti-anxiety drugs, clonazepam (17.2\%) was most commonly prescribed anti-anxiety agent; similar finding was observed in Grover et al. ${ }^{17}$ Benzodiazepines are efficacious for a wide range of conditions such as anxiety, insomnia, tonic-clonic seizures and muscular spasms. In addition, they were widely co-prescribed along with antidepressants and antipsychotics. However, with long term use the adverse effects (memory, impairment, depression, tolerance, dependence) overweigh the benefits, which should be minimized by rational prescribing. Guidelines for the rational use of BZDs recommend their use for short term (maximum four week) or intermittent courses in minimum effective doses, to be prescribed only when symptoms are severe. ${ }^{18}$

Mood stabilizers accounted to be part of $9.80 \%$ prescriptions. A similar result in relation to use of anticonvulsants and mood stabilizers was shown by study of Rode et al. ${ }^{19}$ Sodium valproate was the major mood stabilizer in our study $(8.2 \%)$.

In our study atypical antipsychotics were prescribed to $4.20 \%$ and typical antipsychotics to $23.40 \%$ of patients. Atypical antipsychotics are now rated as first-line agents for the treatment of psychoses because of their low propensity to cause extra pyramidal side-effects (EPS), efficacy against refractory cases and better control against negative symptoms; better tolerance and low relapse rate and safer adverse effect profile. Atypical antipsychotics alleviate both positive and negative symptoms whereas typical antipsychotics can only treat negative symptoms. ${ }^{20}$ However some atypical antipsychotics also show dose related EPS including tardive dyskinesia on long term use, weight gain and hyperprolactinemia. Essential difference between the typical and atypical antipsychotic is the size of therapeutic index in relation to acute EPS. Typical antipsychotics still play an important role in Schizophrenia and offer a valid alternative to atypical where atypical drugs are poorly tolerated. Guidelines of national institute of clinical excellence (NICE) of 2009 suggest that there is no longer any imperative to prescribe an 'atypical' as first line treatment. Clozapine may be offered only after primary failure of two antipsychotic drugs. ${ }^{21}$

Among the atypical antipsychotics, olanzapine was the most commonly prescribed agent; our findings closely match with study of Grover et al. ${ }^{17,22}$ The benefit of olanzapine may be due to its greater efficacy, noticeable improvement of negative symptoms, higher response rate, better maintenance of treatment and finally lower incidence of adverse effects. ${ }^{23}$

The injectable antipsychotics prescribed were flupenthixol and haloperidol. Studies have shown that depot injections are useful in the management of Schizophrenia in acute phases and also for the maintenance treatment. $^{24}$ Concerns about the adverse effects and cost effectiveness of parenteral routes of drug administration, are probably the reason for the low utilization of 'depot injection' formulation in the psychiatry OPD.

The overall prescribing frequency of lithium was $0.8 \%$. Because of low therapeutic index, periodic determination of serum concentrations is crucial. The concern about its narrow therapeutic index and difficulty in obtaining drug levels of lithium, explains the low use of lithium in our centre.

\section{CONCLUSION}

The prescribing pattern can be improved by keeping the number of medicines low; prescribing medicines by generic names, using less parenteral formulations and keeping the cost of therapy low. Such type of study can throw light in promoting, designing policy and motivation of physician for rational use of drugs.

\section{ACKNOWLEDGEMENTS}

Authors would like to thank all the healthcare professionals and the members of Institutional Ethics Committee of VIMSAR, Burla, Odisha for their constant support and guidance.

\section{Funding: No funding sources \\ Conflict of interest: None declared}

Ethical approval: The study was approved by the VIREC (19-I-S-O-149/148)

\section{REFERENCES}

1. American Psychiatric Association. Diagnostic and statistical manual of mental disorders (5th ed.). Washington, DC; 2013. 
2. Murthy R. Mental Health Programme in the 11th five years plan. Indian J Med Res. 2007;11:707-12.

3. Math SB, Chandrashekar CR, Bhugra D. Psychiatric epidemiology in India. Indian $\mathrm{J}$ Med Res. 2007;126(3):183-92.

4. Lee D, Bergmen U. Studies of utilization. In: Strom BC, editor. Pharmacoepidemiology. 1st ed. New York: Churchill Livingstone; 1989:259-73.

5. Hogerzeil HV. Promoting rational prescribing: an international perspective. $\mathrm{Br} \mathrm{J}$ Clin Pharmacol. 1995;39:1-6.

6. Patterson HR. The problems of audit and research. J R Coll Gen Pract. 1986;36:196.

7. Tabish A, Sharma S, Sajid A. Drug utilisation research in psychiatry outdoor in tertiary care hospital, Bastar region. IJPR. 2015;5(4).

8. Thakkar KB, Jain MM, Billa G, Joshi A, Khobragade AA. A drug utilization study of psychotropic drugs prescribed in the psychiatry outpatient department of a tertiary care hospital. J Clin Diagn Res. 2013;7(12):2759-64.

9. Piparva KG, Parmar DM, Singh AP, Gajera MV, Trivedi HR. Drug utilization study of psychotropic drugs in outdoor patients in a teaching hospital.

10. Jorm A, Christensen H, Griffiths K. Changes in depression awareness and attitudes in Australia: the impact of beyondblue: the national depression initiative. Australian New Zealand J Psychiatry. 2006;40:42-6.

11. Goyal D, Munjal S, Gupta D. Drug Utilization Pattern of Psychotropic Drugs Prescribed in the Psychiatric Department of a Tertiary Care Government Hospital, Rajasthan. IOSR J Dental Med Sci. 2016;15(07):80-7.

12. Chandra S, Khan IN, Mateenudin M, Chandrakapure A, Maaz S, Mubin F. Drug utilization study in OPD of a tertiary care hospital in a rural area of Jalna, Maharashtra, India by using WHO prescribing indicators. Int J Basic Clin Pharmacol. 2018;7:55-8.

13. Ajapuje P, Dhengre P, Giri VC, Khakse GM. Drug Prescription Practices among Pediatric Patients in Yavatmal, Central India. Int J Recent Trends Sci Technology. 2012;5(2):104-6.

14. Bajait CS, Pimpalkhute SA, Sontakke SD, Jaiswal KM, Dawri AV. Prescribing pattern of medicine in chronic kidney disease with special emphasis on phosphate binders. IJP. 2014:46:35-9.

15. Mudhaliar MR, Ishrar SMG, Sadubugga P, Narala SR, Chinnakotla V, Yendluri P. Psychotropic drug utilization in psychiatric outpatient department of a teriary care teaching hospital in India. Int J Res Med Sci. 2017;5:1612-6.

16. Wheeler A. Atypical antipsychotic use for adult outpatients in New Zealand's Auckland and Netherland regions. JNZ Med Assoc. 2006;119:1237.

17. Grover S, Avasthi A, Sinha V. Indian Psychiatric Society multicentric study: Prescription patterns of psychotropics in India. Indian $\mathbf{J}$ Psychiatry. 2014;56(3).

18. Ashton H. Guidelines for the rational use of Benzodiazepines. Drugs. 1994;48:25-40.

19. Rode SB, Ajagallay RK, Salankar HV. A study of drug prescribing pattern in psychiatry out-patient department of a tertiary care teaching hospital. Int $\mathbf{J}$ Basic Clin Pharmacol. 2014;3(3):517-22.

20. Serretti A, Ronchi D, Lorenzi C. New antipsychotics and schizophrenia: a review on efficacy and side effects. Curr Med Chem. 2004;11(3):343-58.

21. National Institute for Health and clinical Excellence. Schizophrenia: Core interventions in the treatment and management of schizophrenia in adults in primary and secondary care. Available at: http://www.nice.org.uk/ Accessed on 2 February 2009.

22. Jena M, Mishra S, Mishra SN. Psychotropic drugs prescription pattern in outdoor of tertiary care hospital. Int J Pharm. 2014;4(4):204-8.

23. Tran PV, Hamilton SH, Kuntz AJ. Double-blind comparison of olanzapine versus risperidone in the treatment of schizophrenia and other psychotic disorders. J Clin Psychopharma. 1997;17(5):407-18.

24. Avasthi A, Aggarwal M, Grover S. Research on antipsychotics in India. Indian $\mathbf{J}$ Psychiatry. 2010;52(1):317-40.

Cite this article as: Jena PP, Naik BN, Rath B. Psychotropic drug utilization in psychiatric outpatient department of a tertiary care government hospital. Int J Basic Clin Pharmacol 2020;9:874-8. 